\title{
Incrementally Applied Multifaceted Therapeutic Bundles in Neuroprotection Clinical Trials...Time for Change
}

\author{
W. Andrew Kofke
}

Published online: 10 February 2010

(C) Springer Science+Business Media, LLC 2010

Resolution of complex clinical diseases is an extraordinarily difficult challenge. Using neurocritical care and stroke as one example, and the focus of this commentary, we know that efforts to discover effective neuroprotective therapies have met with only limited success. Over 475 completed clinical trials are listed on the Internet Stroke Trials Registry [1] with few reports of demonstrable efficacy in the acute context. Indeed, Donnan [2] in the 2007 Feinberg stroke lecture of the American Heart Association suggests:

We have reached a stage at which research in this area should stop altogether or radical new approaches adopted.

However, these many negative studies belie the supportive basic laboratory studies that justified the enormous time and expense for such disappointing translational clinical trials. Most of these trials evaluated therapies meant to attenuate single pathways in the pathogenesis of brain damage. Examples of this approach with a partial listing of therapeutic trial targets are presented in Table 1.

This commentary presents concepts that may be an answer to Donnan's plea for a new approach and will:

1. Suggest an explanatory complex biological system model for such reproducible futility thus providing a rationale to suggest that such negative results, in retrospect, are an expected outcome,

W. A. Kofke ( $\square)$

Departments of Anesthesiology and Critical Care, and Neurosurgery, University of Pennsylvania, 7 Dulles Building, 3400 Spruce St., Philadelphia, PA 19104-4283, USA e-mail: kofkea@uphs.upenn.edu
2. Provide support for the notion that only a multimodal approach can reasonably be expected to lead to effective neuroprotective therapies, and

3. Propose a practical research paradigm to develop a multimodal approach which, taken altogether is more likely to produce breakthrough level results that will then be suitable for subsequent multi-institutional evaluation and generalizable implementation.

Moreover, the concepts presented here in the context of neuroprotection, are probably generalizable and applicable to many other complex clinical problems. Certainly, the efficacious use of combination therapy already employed in management of cancer [3] and AIDS [4] provides examples of this approach that might be adapted to neuroprotection.

\section{Impact of Perturbations in Complex Systems}

Single Facet Futility—Why Negative Results, in Retrospect, Are an Expected Outcome

Imagine a factory that makes widgets. A number of wellknown processes are important factors, $F$, for the quality of the final widget as it proceeds, e.g., conveyor speed $\left(F_{1}\right)$, quality of raw material $\left(F_{2}\right)$, bolts $\left(F_{3}\right)$, and steel $\left(F_{4}\right)$, and type of metal used for circuits $\left(F_{5}\right)$. A weighting factor, $W_{i}$, can be applied to each variable, leading to the following circumscribed general equation describing the widget quality, $Q$ :

$Q=W_{1} F_{1}+W_{2} F_{2}+W_{3} F_{3}+W_{4} F_{4}+W_{5} F_{5}$

The important factors are all known and each can be defined with very small variation so any change in any of the factors will produce a reproducible and predictable 
Table 1 Targets of acute stroke therapy trials [1]

\begin{tabular}{lll}
\hline Drugs to improve blood flow & Drugs to protect brain tissue & \\
\hline Anti-thrombotic & Calcium channel blockers & Opioid antagonists \\
Anti-platelet & Calcium chelator & Phosphatidylcholine precursors \\
Fibrinogen depleting & Free radical scavengers_antioxidants & Serotonin agonists \\
Improve capillary flow & GABA agonists & Sodium channel blockers \\
Thrombolytics & Glutamate antagonists & Potassium channel openers \\
Pressors & Growth factors & Mechanism unknown or uncertain \\
& Leukocyte adhesion inhibitors & Nitric oxide inhibitors \\
\hline
\end{tabular}

change in the widget quality. Moreover, when a malfunction arises in one of the system components, the cascade of secondary malfunctions, if any, is relatively straightforward, and a single facet remedy directed to the original problem produces a "cure."

A healthy biological system, in contrast is extraordinarily complex with innumerable interacting processes which in toto produce homeostasis despite minor perturbations. However, a severe injury process, e.g., stroke or traumatic brain injury (TBI), in such a system may overwhelm these homeostatic mechanisms to then lead to dysfunction which can be characterized by outcome severity, $S$. This will result from a pathophysiologically complex injury process, leading to a similar, but openended, descriptive equation being derived with important pathophysiologic factors, $F_{i}$, and weighting factors, $W_{i}$ :

$S=W_{1} F_{1}+W_{2} F_{2}+W_{3} F_{3}+W_{4} F_{4}+W_{5} F_{5} \cdots$

Unlike the broken widget process, however, there are a large number of complex, disparate, and potentially interacting factors known to contribute to $S$ (Fig. 1). There are also an unknown number of as yet unknown factors with correspondingly unknown weighting factors and variability. Moreover, each pathophysiologic factor, $F_{i}$, has to be described over a biologically diverse clinical population such that each factor has an associated central tendency but with a large, possibly non-normal distribution about that mean. Notwithstanding the linear equation model, it seems likely that between-factor interactions may not be simply additive but mathematically more complex, and that some may have a non-linear, exponential impact on severity.

Further adding to the complexity of this pathobiological process, the weighting variable for each factor changes with time after the initial insult, because the importance of various secondary processes wax and wane. For example, the weighting factor for cerebral blood flow impact on outcome severity can be very high early on but becomes less important with time as the weighting factor for reperfusion pathophysiology, initially low, increases with the time after ischemic injury.

Much of the knowledge about pathophysiology and therapy after brain injury is derived from animal models...designed to reduce biological variation, and without complicating concomitant illnesses and pharmacotherapy. Humans, however, present with myriad additional confounding biological and pharmacological factors not included in animal models. In addition, in the clinical environment there are associated system factors, $H_{i}$, such as nursing ratio and experience, drug and technology availability, day of the week and time of day, rapid response team availability and quality, varied standard clinical practices [5], and so on, which also can be important to the eventual injury outcome and likely also lack linearity [6] and with time-dependent variation [7]. Reports of therapeutic success with single institution studies followed by failure in multi-institutional studies, e.g., hypothermia used in traumatic brain injury, is an example of the important impact of system factors and their variation in care [5, 8].
Fig. 1 Multiple processes, interacting and with significant variation contribute to the pathogenesis of brain damage. Unknown and many known processes are not shown

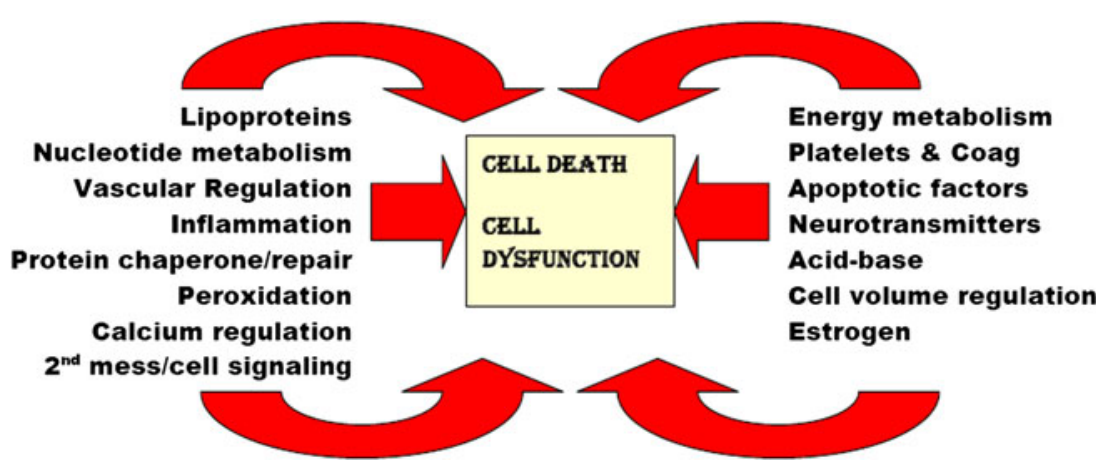


It thus becomes important to include system factors in the variables that contribute to outcome severity after a brain insult. When these system factors and time effects, $t$, and unknown exponents, $e$, for the various factors are added, the entire conceptual severity equation now is rewritten as

$S=\sum W_{i t} F_{i}^{e}+\sum W_{i t} H_{i}^{e}$

These many factors undoubtedly interact with each other such that modification of one may affect others, in a postinsult time-dependent manner. For example, delayed reconstitution of cerebral blood flow may produce changes in other factors associated with reperfusion, e.g., lipid peroxidation or vasogenic edema.

Since there are multiple highly variable and interacting linear and non-linear biological and health system factors that enter into a given outcome, it should come as no surprise that clinical studies designed to ameliorate only one of many complex factors tend to show no or limited therapeutic effect, especially when multi-institutional in design (increasing variation in $H$ ). The exception is when there is truly a breakthrough phenomenon (large $W$, e.g., early thrombolysis in ischemic stroke [9, 10]) or the therapy exerts an intrinsic multifaceted effect (e.g., hypothermia after cardiac arrest [11, 12]).

Conclusion 1: Pathological perturbations of homeostatic mechanisms are not easily corrected by single facet corrective measures in the context of biological complexity.

This leads to the concept that there are serious limitations in the generally accepted use and interpretation of prospective randomized monotherapy studies in the treatment of complex diseases like stroke and TBI such that the medical community has become intellectually paralyzed by traditional scientific notions of knowledge creation. Furthermore, the current widely accepted methods of advancing clinical knowledge for complex problems through single-hypothesis-oriented clinical research may be an unnecessary waste of public resources that has produced innovation paralysis on the part of institutions, third party payers, clinicians, pharma [13], and investigators [2]. An alternate research method that is based on a multifactorial approach to introduce therapeutic bundles which, in aggregate, can be subjected to testing therefore is needed.

Rogalewski et al. [14] and Hemmen and Lyden [15] recently reviewed and endorsed the concept of multifactorial therapy in neuroprotection. Moreover, many authors have demonstrated the feasibility of this approach in creating dramatic levels of neuroprotection in preclinical studies [16-19] and, undoubtedly the multifaceted mechanisms attributed to hypothermia [20] account for its successful translation in cardiac arrest [11, 12] (notably, based on single center studies). None of these authors, however, suggest a rational means of how to introduce the multimodal approach other than trying everything at once...perhaps another prescription for research failure.

Conclusion 2: Multimodal therapy is effective in preclinical neuroprotection research and in other diseases. A rational method is needed to develop multimodal therapeutic bundles in neuroprotection.

\section{PDSA (Plan-Do-Study-Act) Cycles-From QI to Generalizability}

The so-called PDSA method advocated by Berwick [21] for local quality improvement may provide a technique to develop an incrementally implemented multifaceted approach to serially test and add single clinically unproven but safe, pathophysiologically sensible, and otherwise scientifically supported facets in the therapy of a complex disease. Such an approach should produce a multi faceted therapeutic bundle that then may be amenable to more widespread testing and/or application.

The PDSA method usually entails application of a nonrandomized process, using institutional and individual (paired or $N$ of 1) historical control data, to introduce incremental improvements in processes of care. The first step, planning, entails identification of a process to improve with a plan for implementation. The do phase entails the actual systematic implementation of a new process of care. Studying then is the procedure for collecting and analyzing the results of the new intervention. The act phase entails coming to a conclusion that the results have merit or not and then to produce a permanent yet incremental change in a system's health care protocols.

The PDSA technique is designed for processes of therapeutic QI that are meant to improve process-of-care outcomes locally and not necessarily produce generalizable knowledge. Thus, by the federal definition of the United States such processes technically are not research. Nonetheless, the PDSA process is a widely accepted type of institutional process-of-care experimentation which very well could be used as a method to produce or contribute to the production of new generalizable knowledge for complex biological diseases.

\section{Using PDSA Cycles to Develop a Multifaceted Program to Arrest Secondary Brain Damage}

These notions lead to the proposed concept to incrementally introduce therapeutic modalities, each individually directed against single facets in known processes of 
secondary injury after a neurologic insult, followed sequentially by three primary measurements:

1. Impact on the target pathophysiologic pathway.

2. Surrogate neurologic outcome measures.

3. Functional neurologic measures.

Chosen pathophysiologic goals should be widely accepted to have neuroprotective or neuroregenerative and recovery potential based on either animal or clinical studies. A specific therapy should be first shown to safely produce an appropriate pathophysiologic goal (e.g., decrease oxidation due to free radicals). This should be followed by surrogate measurements of neurologic efficacy (e.g., biomarkers [22], magnetic resonance imaging/ spectroscopy, or cranial computed tomographic evaluation) and by subsequent measurements of clinically meaningful outcome. The general decision flow diagram is illustrated in Fig. 2.

For example, a fictional research team decides they want to develop a therapeutic bundle designed to attenuate brain damage after cardiac arrest. The group studies previously published research and decide to target the following pathophysiologic contributors with the noted therapeutic interventions and evaluations of pathophysiologic efficacy:

- Decreased cerebral blood flow post ischemia

- Hypertensive therapy [23]

- Measure blood pressure

- Measure CBF

- Anticoagulation with heparin [17]

- Measure partial thromboplastin time

- Measure CBF

- Modest hemodilution [17]

- Measure hematocrit

- Measure CBF

- Thromboxane inhibitors [24]

- Measure CBF

- Impaired ATP production during resuscitation and after return of spontaneous circulation

- Near infra-red transcranial laser light therapy [25]

- Measure ATP and phosphocreatine with magnetic resonance spectroscopy

- Reperfusion related free radical injury

- NXY-059 [26]

- Measure plasma and urine isoprostanes

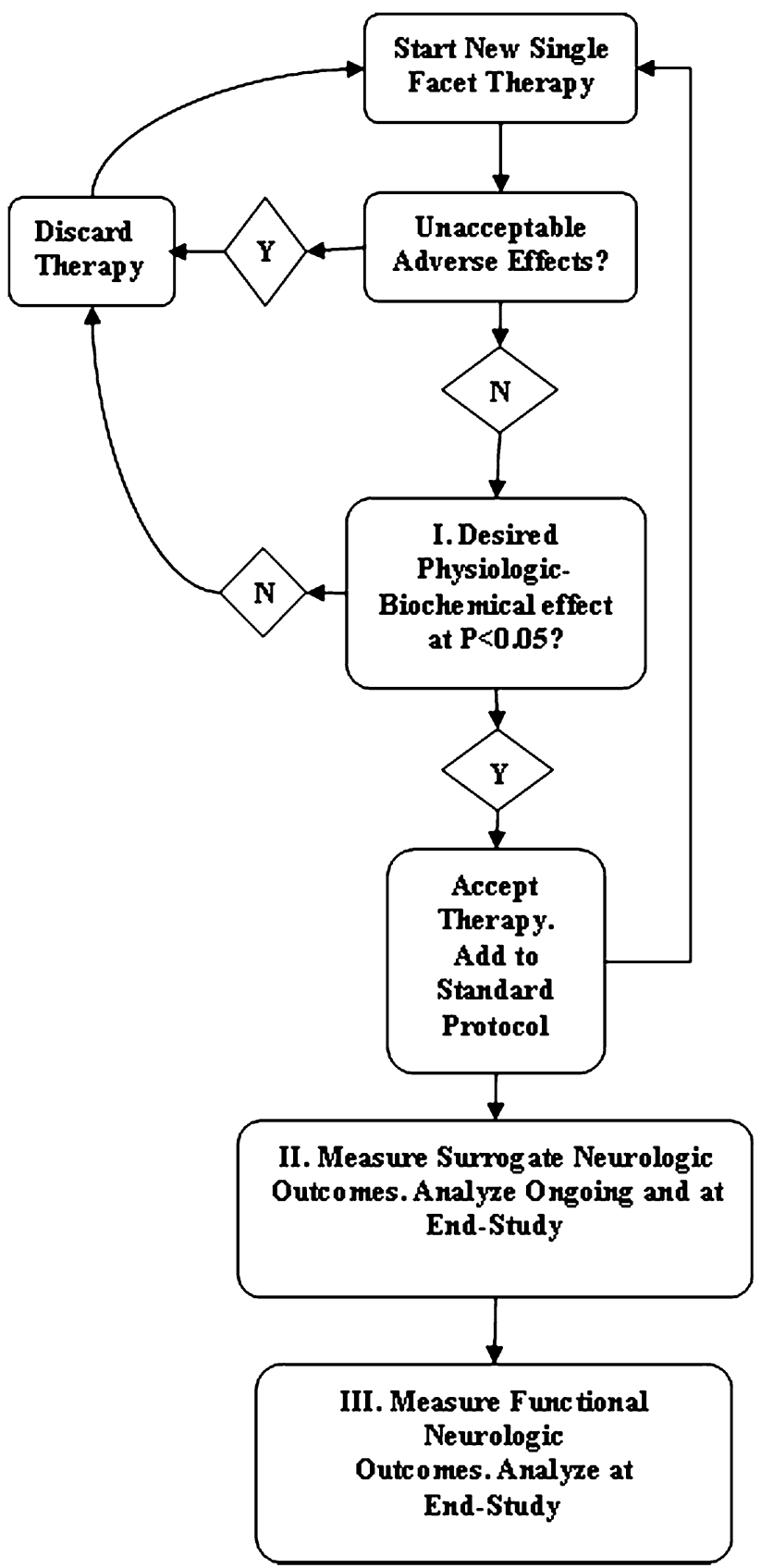

Fig. 2 PDSA cycle to serially evaluate and implement components of a neuroprotective therapeutic bundle

- Necroptosis [27]

- Necroptosis inhibitor

- Caspase independent serum biomarker [28]

- Increased intracellular calcium

- Dantrolene [29]

- Measure plasma dantrolene concentration 
- Mitochondrial permeability transition pore opening

- Cyclosporine [30]

- Measure plasma or CSF cyclosporine concentration

- Apoptosis

- Granulocyte colony stimulating factor [31]

- Measure calpain associated biomarkers [28]

- Glutamate associated injury

- Ketamine and barbiturate infusion [32]

- Measure plasma ketamine and barbiturate concentrations

- EEG to evaluate brain effect

- Brain catecholamine associated injury

- Propranolol [33]

- Measure plasma catecholamines

- Reserpine [34]

- Measure plasma catecholamines

Each therapeutic intervention is added singly, safety is confirmed, pathophysiologic efficacy is confirmed, and surrogate and functional measures of neuroprotective efficacy are monitored throughout the study, which may take many years to complete. At the end of the project outcome data such as shown in Fig. 3 should be apparent. Note that this is a fictional group of proposed therapies for the sole purpose of illustrating the use of PDSA in developing a neuroprotective therapeutic bundle. Those more immersed in researching this disease would undoubtedly propose more suitable therapeutic targets to evaluate.
Evidence supporting the utility of this approach may be derived from observations that implementation of the "therapeutic bundle" advocated by the Brain Trauma Foundation is associated with a dramatic improvement in outcome after traumatic brain injury [35]. Notably, the individual facets neither have simultaneously developed nor have they been devised in a coordinated manner. This is said to be evidence based; however, only one of the 14 recommendations is based on level one evidence and five are based on level three or less evidence. This contrasts with the relatively unchanging morbidity and mortality over recent decades [36, 37] after intracerebral hemorrhage, a disease which has not been subjected to the same multifaceted therapy as has occurred with traumatic brain injury.

Conclusion 3: The PDSA method works well to serially and incrementally improve local processes of care and should be adaptable as a method to develop generalizable multimodal therapeutic bundles for neuroprotection.

Implementation of this proposed paradigm will have expected and undoubtedly also unforeseen obstacles. Some of the foreseeable problems follow:

- It may be impossible to obtain timely information regarding the pathophysiologic efficacy of a given therapy. For example, a drug directed at altering a brain neurotransmitter would require invasive brain microdialysis data to really show the effect. This may not be ethically justifiable.

- It is unclear the best way, statistically to show pathophysiologic and surrogate outcome efficacy. One issue, as presented, is the use of internal historical controls. Such an approach introduces the well-known possibility of bias due to unmeasured unknown processes that may

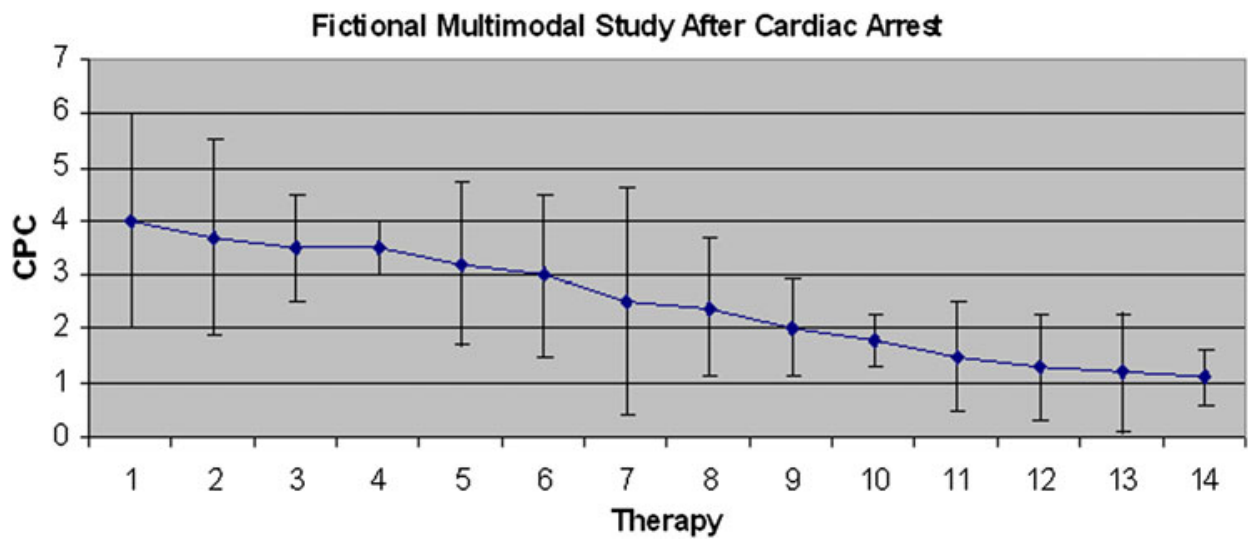

Fig. 3 Possible results over time with incremental additions of neuroprotective therapies. CPC is clinical performance category but it could also be levels of serum biomarkers for brain damage, magnetic resonance spectroscopy data, or other surrogate and functional neurologic outcome measures. Note that sequential addition of each therapy has a variation that would suggest no immediate statistically significant effect but that taken in aggregate over time a statistically significant effect should be able to be demonstrated 
systematically change over time within an institution. An alternate design would call for an untreated control group which would undergo the same surrogate and functional outcome measures. Given that the intent is simply to efficiently create a therapeutic bundle for later comparison with an untreated control group, this approach may not be necessary, but nonetheless certainly a reasonable alternative.

- This model, as currently constructed, provides for no appropriate dose-finding methodology. This is a relatively new and developing area in design of clinical research studies and, conceptually, such techniques might be included in the PDSA approach, but using pathophysiologic endpoints, where available, as the goal in dose finding.

- Sample size calculation may be problematic. Pathophysiologic endpoints probably could be used with serial assessment during the study for the likelihood and minimization of type I and II errors. By the end of therapeutic bundle development there should be enough preliminary data available on clinically meaningful outcomes to then be able to estimate sample sizes for a subsequent properly conducted prospective randomized trial of the therapeutic bundle versus an untreated control group.

- Interactions between therapies may arise. This may be unavoidable and hopefully the interactions will both be in a positive direction, although this may not necessarily arise. It seems the best approach would be to monitor for interactions by continually measuring pathophysiologic endpoints for previously accepted therapies. For example, inclusion of an antioxidant in the accepted paradigm should then mandate continued monitoring for isoprostanes as new therapies are added.

- It is unclear how many modes to test within a given therapeutic bundle nor the order in which to serially evaluate them. Ideally modes are serially added until a statistically significant clinically meaningful effect is observed. Moreover, given a desire for efficient use of resources, the planners should seek to test and implement modes earlier that are expected to have higher impact. However, translation to testing in a broader multi-institutional context will increase heterogeneity such that a higher level of statistical significance may be first sought before going to a prospective randomized multi-institutional evaluation of the therapeutic bundle.

- Finally, is it really necessary to introduce each facet singly? Perhaps bundles that are directed to a single goal could be added simultaneously in a goal directed manner [38].

By the end of the development of a given overall therapeutic bundle, multiple new neuroprotective therapies (versus secondary injury) will have been added to the neuroprotective cocktail as part of an IRB overseen PDSA process. A robust difference in surrogate and functional outcomes should be apparent compared with historical controls. The results can then be offered in aggregate as a multifaceted therapeutic bundle ready for multi-institutional trial or...for a true breakthrough effect...possibly for immediate generalized implementation.

Although not satisfying in a purely scientific sense, this approach may nonetheless offer the potential to introduce effective breakthrough therapy for acute neurologic syndromes that heretofore have proven recalcitrant to solutions using standard scientific methods. As such, the potential benefits seem to outweigh possible risks.

Acknowledgments This was presented at the 2008 meeting of the Society of Neurologic Anesthesia and Critical Care and the 2009 meeting of the Neurocritical Care Society. The author gratefully acknowledges the helpful comments of Peter LeRoux and Sherman Stein in the development of the manuscript. The author has no conflicts of interest.

\section{References}

1. Stroke Trials Registry. 2008. http://www.strokecenter.org/trials/ index.aspx. Accessed 6 January 2009.

2. Donnan GA. The 2007 Feinberg lecture: a new road map for neuroprotection. Stroke. 2008;39(1):242.

3. Chura JC, Marushin R, Boyd A, Ghebre R, Geller MA, Argenta PA. Multimodal therapy improves survival in patients with CNS metastasis from uterine cancer: a retrospective analysis and literature review. Gynecol Oncol. 2007;107(1):79-85.

4. Bartlett JA, Fath MJ, Demasi R, et al. An updated systematic overview of triple combination therapy in antiretroviral-naive HIVinfected adults. AIDS. 2006;20(16):2051-64. (see comments).

5. Clifton G, Choi S, Miller E, et al. Intercenter variance in clinical trials of head trauma-experience of the National Acute Brain Injury Study: Hypothermia. J Neurosurg. 2001;95(5):751-5.

6. Strum DP, May JH, Vargas LG. Modeling the uncertainty of surgical procedure times: comparison of log-normal and normal models. Anesthesiology. 2000;92(4):1160-7.

7. Moore IC, Strum DP, Vargas LG, Thomson DJ. Observations on surgical demand time series: detection and resolution of holiday variance. Anesthesiology. 2008;109(3):408-16.

8. Peterson K, Carson S, Carney N. Hypothermia treatment for traumatic brain injury: a systematic review and meta-analysis. J Neurotrauma. 2008;25(1):62-71.

9. Albers GW, Amarenco P, Easton JD, Sacco RL, Teal P. Antithrombotic and thrombolytic therapy for ischemic stroke: American College of Chest Physicians Evidence-Based Clinical Practice Guidelines (8th Edition). Chest. 2008;133(6 Suppl): 630S-69S.

10. Wardlaw JM, del Zoppo G, Yamaguchi T. Thrombolysis for acute ischaemic stroke. Cochrane Database Syst Rev. 2000;(2): CD000213 (see comment update in Cochrane Database Syst Rev. 2003;(3):CD000213; PMID: 12917889).

11. Group HaCAS. Mild therapeutic hypothermia to improve the neurologic outcome after cardiac arrest. N Engl J Med. 2002; 346(8):549-56. 
12. Bernard SA, Gray TW, Buist MD, et al. Treatment of comatose survivors of out-of-hospital cardiac arrest with induced hypothermia. N Engl J Med. 2002;346(8):557-63.

13. Feuerstein GZ, Chavez J. Translational medicine for stroke drug discovery: the pharmaceutical industry perspective. Stroke. 2009; 40(3 Suppl):S121-5.

14. Rogalewski A, Schneider A, Ringelstein EB, Schabitz WR. Toward a multimodal neuroprotective treatment of stroke. Stroke. 2006;37(4):1129-36.

15. Hemmen TM, Lyden PD. Multimodal neuroprotective therapy with induced hypothermia after ischemic stroke. Stroke. 2009; 40(3 Suppl):S126-8.

16. Zausinger S, Scholler K, Plesnila N, Schmid-Elsaesser R. Combination drug therapy and mild hypothermia after transient focal cerebral ischemia in rats. Stroke. 2003;34(9):2246-51.

17. Safar P, Stezoski W, Nemoto EM. Amelioration of brain damage after 12 minutes' cardiac arrest in dogs. Arch Neurol. 1976;33(2): 91-5.

18. Aronowski J, Strong R, Shirzadi A, Grotta JC. Ethanol plus caffeine (caffeinol) for treatment of ischemic stroke: preclinical experience. Stroke. 2003;34(5):1246-51.

19. Culmsee C, Junker V, Kremers W, Thal S, Plesnila N, Krieglstein J. Combination therapy in ischemic stroke: synergistic neuroprotective effects of memantine and clenbuterol. Stroke. 2004; 35(5):1197-202.

20. Sahni R, Sanocka UM. Hypothermia for hypoxic-ischemic encephalopathy. Clin Perinatol. 2008;35(4):717-34.

21. Berwick DM. Developing and testing changes in delivery of care. Ann Intern Med. 1998;128(8):651-6.

22. Siman R, Zhang C, Roberts VL, Pitts-Kiefer A, Neumar RW. Novel surrogate markers for acute brain damage: cerebrospinal fluid levels correlate with severity of ischemic neurodegeneration in the rat. J Cereb Blood Flow Metab. 2005;25(11):1433-44.

23. Sterz F, Leonov Y, Safar P, Radovsky A, Tisherman SA, Oku K. Hypertension with or without hemodilution after cardiac arrest in dogs. Stroke. 1990;21(8):1178-84.

24. Itoh Y. Blockade of thromboxane A2 receptor ameliorates delayed postischemic hypoperfusion of the brain in cats. Keio $\mathrm{J}$ Med. 1994;43(2):88-93.

25. Zivin JA, Albers GW, Bornstein N, et al. Effectiveness and safety of transcranial laser therapy for acute ischemic stroke. Stroke. 2009;40(4):1359-64.

26. Lees KR, Zivin JA, Ashwood T, et al. NXY-059 for acute ischemic stroke. N Engl J Med. 2006;354(6):588-600. (see comment).
27. Hitomi J, Christofferson $\mathrm{DE}, \mathrm{Ng} \mathrm{A}$, et al. Identification of a molecular signaling network that regulates a cellular necrotic cell death pathway. Cell. 2008;135(7):1311-23.

28. Siman R, McIntosh TK, Soltesz KM, Chen Z, Neumar RW, Roberts VL. Proteins released from degenerating neurons are surrogate markers for acute brain damage. Neurobiol Dis. 2004; 16(2):311-20.

29. Nakayama R, Yano T, Ushijima K, Abe E, Terasaki H. Effects of dantrolene on extracellular glutamate concentration and neuronal death in the rat hippocampal CA1 region subjected to transient ischemia. Anesthesiology. 2002;96(3):705-10.

30. Sinigaglia-Coimbra R, Cavalheiro EA, Coimbra C. Protective effect of systemic treatment with cyclosporine A after global ischemia in rats. J Neurol Sci. 2002;203-204:273-6.

31. Lu CZ, Xiao BG. G-CSF and neuroprotection: a therapeutic perspective in cerebral ischaemia. Biochem Soc Trans. 2006; 34(Pt 6):1327-33.

32. Shibuta S, Varathan S, Mashimo T. Ketamine and thiopental sodium: individual and combined neuroprotective effects on cortical cultures exposed to NMDA or nitric oxide. Br J Anaesth. 2006;97(4):517-24.

33. Neil-Dwyer G, Walter P, Cruickshank J. Beta-blockade benefits patients following a subarachnoid hemorrhage. Eur J Clin Pharmacol. 1985;28:25.

34. Busto R, Harik S, Yoshida S, Scheinberg P, Ginsberg M. Cerebral norepinephrine depletion enhances recovery after brain ischemia. Ann Neurol. 1985; 18:329.

35. Fakhry SM, Trask AL, Waller MA, Watts DD. Management of brain-injured patients by an evidence-based medicine protocol improves outcomes and decreases hospital charges. J TraumaInjury Infect Crit Care. 2004;56(3):492-9. discussion 499-500.

36. Flaherty ML, Haverbusch M, Sekar P, et al. Long-term mortality after intracerebral hemorrhage. Neurology. 2006;66(8):1182-6.

37. Manno EM, Atkinson JL, Fulgham JR, Wijdicks EF. Emerging medical and surgical management strategies in the evaluation and treatment of intracerebral hemorrhage. Mayo Clin Proc. 2005; 80(3):420-33.

38. Rivers E, Nguyen B, Havstad S, et al. Early goal-directed therapy in the treatment of severe sepsis and septic shock. N Engl J Med. 2001;345(19):1368-77. (see comment). 